\title{
Synovial chondromatosis and soft tissue chondroma: extraosseous cartilaginous tumor defined by FN1 gene rearrangement
}

\author{
Fernanda Amary $\mathbb{( I D}^{1} \cdot$ Luis Perez-Casanova ${ }^{1} \cdot$ Hongtao $\mathrm{Ye}^{1} \cdot$ Lucia Cottone $^{2} \cdot$ Anna-Christina Strobl $^{1} \cdot$ Paul Cool $\mathbb{D}^{3}$. \\ Elena Miranda ${ }^{2}$ - Fitim Berisha ${ }^{1} \cdot$ William Aston $^{1} \cdot$ Maia Rocha ${ }^{1,2} \cdot$ Paul O'Donnell $^{1} \cdot$ Nischalan Pillay $^{1,2}$. \\ Roberto Tirabosco ${ }^{1}$ Daniel Baumhoer $\mathbb{D}^{4} \cdot$ Edward S. Hookway ${ }^{2} \cdot$ Adrienne M. Flanagan $^{1,2}$
}

Received: 31 March 2019 / Revised: 4 June 2019 / Accepted: 4 June 2019 / Published online: 4 July 2019

(c) The Author(s), under exclusive licence to United States \& Canadian Academy of Pathology 2019

\begin{abstract}
A fusion between fibronectin $1(F N 1)$ and activin receptor 2A (ACVR2A) has been reported previously in isolated cases of the synovial chondromatosis. To analyze further and validate the findings, we performed FISH and demonstrated recurrent FN1-ACVR2A rearrangements in synovial chondromatosis (57\%), and chondrosarcoma secondary to synovial chondromatosis (75\%), showing that FN1 and/or AVCR2A gene rearrangements do not distinguish between benign and malignant synovial chondromatosis. RNA sequencing revealed the presence of the $F N 1-A C V R 2 A$ fusion in several cases that were negative by FISH suggesting that the true prevalence of this fusion is potentially higher than $57 \%$. In soft tissue chondromas, FN1 alterations were detected by FISH in $50 \%$ of cases but no ACVR2A alterations were identified. RNA sequencing identified a fusion involving $F N 1$ and fibroblast growth factor receptor 2 (FGFR2) in the case of soft tissue chondroma and FISH confirmed recurrent involvement of both FGFRI and FGFR2. These fusions were present in a subset of soft tissue chondromas characterized by grungy calcification, a feature reminiscent of phosphaturic mesenchymal tumor. However, unlike the latter, fibroblast growth factor 23 (FGF23) mRNA expression was not elevated in soft tissue chondromas harboring the FNI-FGFRI fusion. The mutual exclusivity of ACVR2A rearrangements observed in synovial chondromatosis and FGFRI/2 in soft tissue chondromas suggests these represent separate entities. There have been no reports of malignant soft tissue chondromas, therefore differentiating these lesions will potentially alter clinical management by allowing soft tissue chondromas to be managed more conservatively.
\end{abstract}

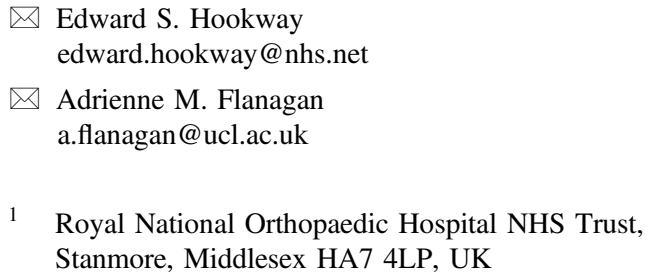

1 Royal National Orthopaedic Hospital NHS Trust, Stanmore, Middlesex HA7 4LP, UK

These authors contributed equally: Fernanda Amary, Luis PerezCasanova

These authors contributed equally: Edward S Hookway, Adrienne M Flanagan

Supplementary information The online version of this article (https:// doi.org/10.1038/s41379-019-0315-8) contains supplementary

material, which is available to authorized users.

\section{Introduction}

Synovial chondromatosis is a rare benign neoplasm associated with the synovium of a joint, tendon sheath, or bursa. The presence of endochondral ossification in up to $50 \%$ of cases led to the synonym synovial osteochondromatosis [1]. Any synovial joint can be involved, but it is more common in larger joints including the knee, hip, and elbow. The lesion presents most commonly over 40 years of age and there is a male predominance (2:1). Histologically, synovial chondromatosis is characterized by multiple hyaline

2 Cancer Institute, 72 Huntley Street, University College London, London WC1E 6BT, UK

3 The Robert Jones and Agnes Hunt Orthopaedic Hospital NHS Foundation Trust, Oswestry SY10 7AG, UK

4 Bone Tumor Reference Center, Institute of Pathology, University Hospital Basel, Basel, Switzerland 
cartilage nodules intimately related to the synovium. These may be embedded within the subsynovial tissue but may also lie in the synovial fluid either attached to the synovium or as 'floating' bodies.

The recent findings of $F N 1-A C V R 2 A$ and $A C V R 2 A-F N 1$ in-frame fusions in a case of chondrosarcoma arising secondary to synovial chondromatosis and in one of seven cases of synovial chondromatosis [2], indicate that these lesions represent neoplasms, and that they rarely transform into secondary chondrosarcoma [3-6]. However, the findings by Totoki et al., to the best of our knowledge, have not yet been validated. Since peripheral and central chondrosarcoma, chondroblastoma, phosphaturic mesenchymal tumor, and extraskeletal mesenchymal chondrosarcoma are among the potential differential diagnoses of synoviumrelated cartilage tumors, a reliable biomarker could aid significantly in the diagnostic accuracy of these lesions, particularly in needle core biopsies [7-10].

Soft tissue chondromas occur most commonly in the hands and feet and share histological features with synovial chondromatosis, hence it is one of the main differential diagnoses at these sites. Such lesions are considered to represent distinct entities [11-14] and soft tissue chondromas are by definition not related to the synovial lining. Nevertheless, even with the use of high-resolution radiological imaging in individual cases it can be practically impossible to exclude unequivocally an association with a tendon sheath, a bursa lining, or synovium in the hands and feet. A specific biomarker would therefore aid in rendering the correct diagnosis [15].

In this study we set out to determine the prevalence of FN1 and ACVR2A gene rearrangements in a larger set of synovial chondromatosis, and secondary synovial chondrosarcoma, and to search for recurrent genetic alterations in soft tissue chondromas.

\section{Materials and methods}

Cases were identified by searching the histopathology files at the Royal National Orthopaedic Hospital (RNOH), UK, The Robert Jones and Agnes Hunt Orthopaedic Hospital (RJAH), and the Basel Bone Tumor Reference Centre, Switzerland. The RNOH Biobank was approved by the National Research Ethics Committee of the Health Research Committee (reference 15/YH/0311: Integrated Research Application System (IRAS) project identifier: 18309). This specific project was approved by the National Research Ethics Committee approved UCL/ UCLH Biobank Ethics Committee (specific project reference no. EC17.14). This biobank was licensed by the Human Tissue Authority under number 12073. Cases from the RJAH biobank were approved by the National Research Ethics Committee of the Health Research Committee (reference 17/YH/0108; IRAS project identifier: 217446 and licensed by the HTA under number 12073). Ethical approval was also given by the Ethikkommission bei der Basel (reference 274/12).

All samples were diagnosed by specialist bone tumor pathologists (AMF, DB, FA, and RT) according to the World Health Organization classification 2013 [13, 14]. When available, cases were correlated with clinical and imaging data. All samples were fixed in $10 \%$ formal saline, decalcified in EDTA or in nitric acid (5\%), and processed in paraffin: tissue sections were stained with haematoxylin and eosin.

\section{Fluorescence in situ hybridization (FISH)}

FISH was carried out as described previously [16]. In brief, sections were marked by a specialist histopathologist to target tumor-rich area, then deparaffinized and pretreated by pressure-cooking following incubation in pepsin solution at $37^{\circ} \mathrm{C}$ for $50 \mathrm{~min}$. Probes were added to tissue sections, denatured at $72{ }^{\circ} \mathrm{C}$, and hybridized overnight at $37^{\circ} \mathrm{C}$. Thereafter, the sections were washed and counterstained with 4', 6-diamidino-2-phenylindole (DAPI) and mounted with coverslips.

Probes used for FISH included Agilent SureFISH custom-designed $F N 1$ and $A C V R 2 A$ break-apart probes (Agilent, Cheshire, UK) (Table S1) and commercially available break-apart probes for FGF1 (CytoTest, Rockville, USA), FGFR1 (Leica Biosystems, Wetzlar, Germany), fibroblast growth factor receptor 2 (FGFR2) (ZytoVision, Bremerhaven, Germany), and p16/CDKN2A (9p21) (Vysis, Abbott Molecular, IL, USA). A positive result was obtained when at least $15 \%$ of the nuclei analyzed reveal an aberrant signal on counting a minimum of 50 consecutive nonoverlapping nuclei. Assessment of $p 16 /$ $C D K N 2 A$ FISH was undertaken as previously reported [17]. Samples previously known to have rearrangement for FGFR1, FGF1, and FGFR2 by FISH and/or RNA sequencing were employed as controls.

\section{RNAscope}

The expression of FGF23 was assessed using the RNAscope $^{\mathrm{TM}}$ assay (Advanced Cell Diagnostics, Hayward, CA, USA) according to the manufacturer's instructions. Sections were hybridized with a probe specific for FGF23 (HsFGF23, Cat. 557241), and for the endogenous control Ubiquitin C (Hs-UBC, Cat. 310041). Hybridization signals were amplified and visualized with the RNAscope $2.5 \mathrm{HD}$ Detection Kit (Brown; Cat. 322360). 


\section{Quantitative real time PCR (qPCR)}

RNA was extracted from FFPE sections using the Ambion RecoverAll ${ }^{\mathrm{TM}}$ Total Nucleic Acid Isolation kit (Ambion, Austin, TX, USA) and reverse transcribed into cDNA with the Invitrogen $^{\mathrm{TM}}$ SuperScript ${ }^{\mathrm{TM}}$ IV First-Strand Synthesis System with random hexamers (Thermo Fisher Scientific, Loughborough, Leicestershire, UK) according to manufacturer's instructions. qPCR was carried out using TaqMan ${ }^{\circ}$ Gene Expression Assays (Thermo Fisher Scientific): FGF23 (Cat. Hs00221003_m1), and the endogenous control G6PD (Cat. Hs00166169_m1). For analysis, the means of cycle thresholds $(\mathrm{Ct})(n=2)$ were calculated. Based on the $\Delta \mathrm{Ct}$ between the gene and G6PD, the fold increase of gene expression was determined.

\section{RNA extraction, library preparation, RNA sequencing}

RNA sequencing was performed on cases that were retrieved from the operating theaters immediately after excision. A portion from each of the tumors was snap frozen. Frozen sections were cut and those that were at least $60 \%$ tumor-rich were homogenized in $1 \mathrm{ml}$ Trizol followed by extraction using the DirectZol purification kit (Zymo Research, Irvine, CA, USA) according to the manufacturer's instructions. RNA quality was assessed on the TapeStation 2200 (Agilent, Santa Clara, CA, USA): a RIN value of greater than seven was obtained in each case. Poly (A) RNA was selected from 200 ng total RNA using the NEBNext Poly(A) mRNA selection module (NEB, Ipswich, MA, USA), and first-strand libraries constructed with the NEBNext Ultra Directional RNAseq library preparation kit (NEB) according to the manufacturer's instructions. Libraries were pooled and sequenced with $2 \times 101$ bp reads on a Hiseq 1500 (Illumina, San Diego, CA, USA) in rapid run mode. Fusion genes were identified from sequenced RNA with Fusion Catcher using default settings with Bowtie, BLAT, and STAR used as the aligners and GRCh37 as the reference genome [18].

\section{Immunohistochemistry}

Immunohistochemistry was performed in the Leica Bond 3 fully automated immunohistochemistry stainer (Leica
Microsystems, Milton Keynes, UK) using the rabbit monoclonal histone H3F3 K36M mutant antibody (clone RM193; RevMAb Biosciences USA, San Francisco, CA, USA). Leica epitope retrieval 2 solution was used for 20 min. The antibody was diluted 1:400 [8].

\section{Results}

One-hundred and sixteen samples from the same number of patients were analyzed by FISH for $F N 1$ and $A C V R 2 A$ gene rearrangement including 90 synovial chondromatoses, seven synovial chondrosarcomas, and 19 soft tissue chondromas. Of these, 80 samples $(69 \%)$ gave informative results (Tables 1 and S2). The percentage of break-apart samples in this study varied from 20 to $80 \%$ of the cells in the cases in which a rearrangement was found.

Synovial chondromatosis $(n=90)$ showed an almost equal gender ratio $(1.14 \mathrm{M}: 1 \mathrm{~F})$ and presented between the ages of nine and 94 years of age (mean 48). The knee was the most frequently affected site (47 cases, $52 \%$ ) followed by hand/wrist [11], hip [11], elbow [8], foot/ankle [8], and shoulder [5]. Tumor size was available from 57 patients and ranged from 4 to $170 \mathrm{~mm}$ (mean $49 \mathrm{~mm}$ ). Of the $40 / 89$ patients whose tumors were resected and for whom followup information was available, $15(38 \%)$ developed a local recurrence between 6 and 236 months (mean 45 months). Three of these 15 patients developed a second recurrence at 11,12 , and 90 months following surgery for their first relapse. The follow-up period of the 25 patients $(63 \%)$ who did not develop a recurrence ranged from 1 to 57 months (mean 18 months).

Histological assessment revealed multinodular tumors, connected to the synovium and composed of chondrocytes often arranged in clusters (clonal pattern of arrangement) with mild to moderate variation in cell size. The matrix frequently demonstrated enchondral ossification and/or calcification to a varying extent. No histological differences were detected in cases with or without gene rearrangement (Fig. 1).

Of the 90 synovial chondromatosis, 58 gave informative FISH results for $F N 1$ and/or ACVRA2 gene rearrangements (Table 1, Table S2), and 57\% (33/58) of which revealed a

Table 1 Rearrangement of FN1 and ACVR2A

\begin{tabular}{lccccc}
\hline & $F N 1$ and $A C V R 2 A$ rearrangement & & No rearrangement \\
\cline { 2 - 5 } & Any FN1/ACVR2A rearrangement & Both FN1 and ACVR2A & FN1 only & $A C V R 2 A$ only \\
\hline Synovial chondromatosis $(n=58)$ & $33(56.9 \%)$ & 11 & 8 & 14 & $25(43.1 \%)$ \\
Synovial chondrosarcoma $(n=4)$ & $3(75 \%)$ & 1 & 1 & 1 & $1(25 \%)$ \\
Soft tissue chondroma $(n=18)$ & $9(50 \%)$ & 0 & 9 & 0 & $9(50 \%)$ \\
\hline
\end{tabular}


Fig. 1 Synovial chondromatosis of the left knee. Lateral radiograph showing a large calcified mass in the posterior aspect of the left knee, with small calcific foci anteriorly. Photomicrograph (bottom image) showing the classical multinodular pattern of synovial chondromatosis with areas of calcification and endochondral ossification. FISH analysis shows break-apart signals using $A C V R 2 A$ probe (top right)

Fig. 2 Graphic representation of the gene rearrangement type by site of disease in cases of synovial chondromatosis

Table 2 Characteristics of synovial chondrosarcoma cases
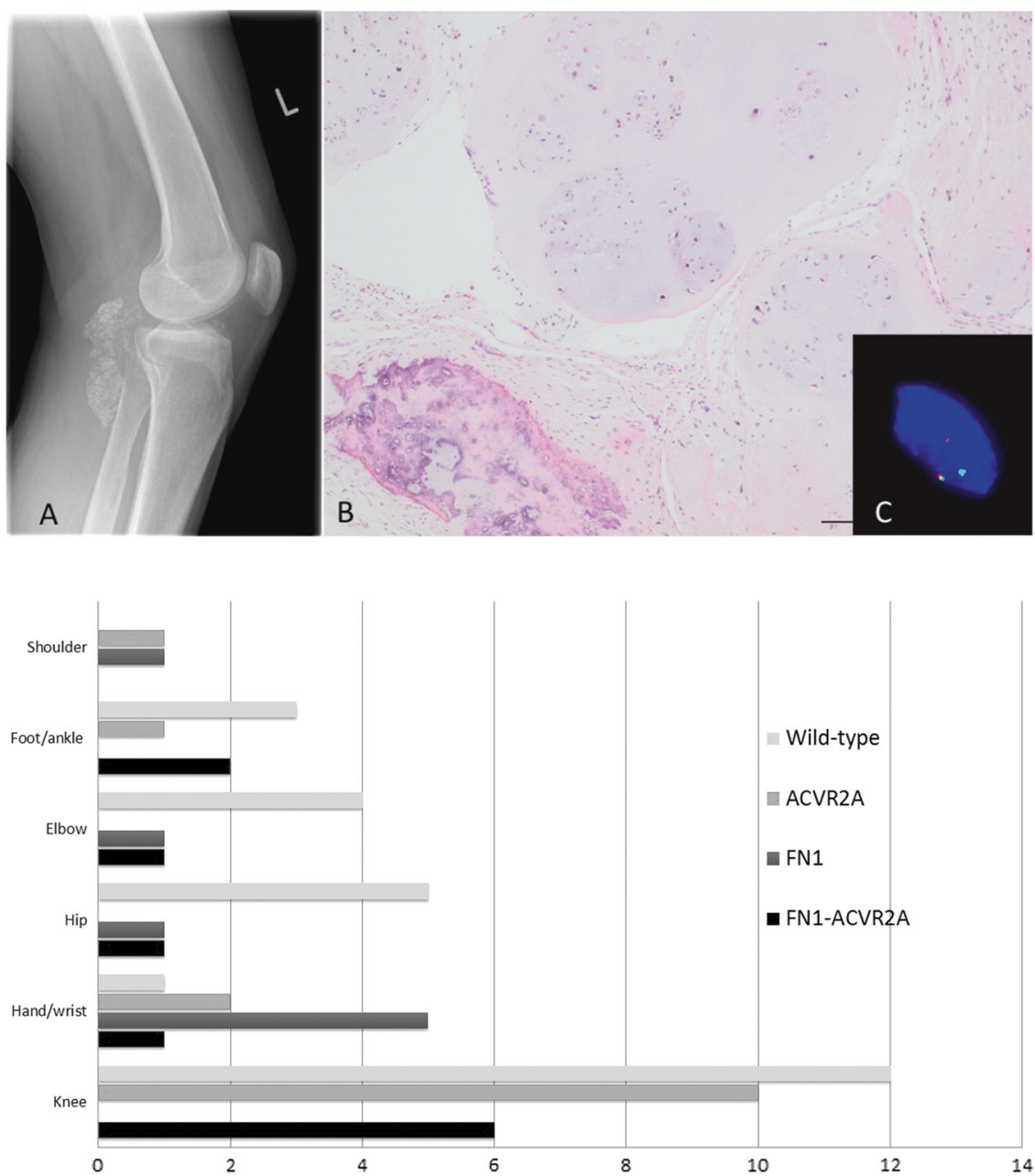

\begin{tabular}{|c|c|c|c|c|c|c|c|}
\hline \multirow[t]{2}{*}{ Case } & \multirow[t]{2}{*}{ Site } & \multirow[t]{2}{*}{ Grade } & \multirow[t]{2}{*}{ Primary/local recurrence } & \multirow[t]{2}{*}{ FN1 } & \multicolumn{2}{|l|}{ FISH } & \multirow[t]{2}{*}{ Clinical outcome } \\
\hline & & & & & $A C V R 2 A$ & $C D K N 2 A$ & \\
\hline 1 & Hand & III & Primary & + & - & Homozygous deletion $^{c}$ & Lost to FU \\
\hline 2 & Knee & II & Primary $^{\mathrm{a}}$ & + & + & Homozygous deletion & Died of disease \\
\hline 3 & Wrist & I & Local recurrence & - & - & Disomic* & Disease free \\
\hline 4 & Hip & III & Local recurrence & NI & NI & NI & Died of disease \\
\hline 5 & Elbow & II & Local recurrence $^{\mathrm{b}}$ & NI & NI & NI & Lost to FU \\
\hline 6 & Hip & II & Local recurrence $^{\mathrm{b}}$ & NI & NI & NI & Lost to FU \\
\hline 7 & Knee & III & Primary & - & + & Copy number gain & Disease free \\
\hline
\end{tabular}

Asterisk represents FISH performed on benign synovial chondromatosis; tissue for FISH was not available from the malignant synovial chondrosarcoma

${ }^{a}$ Histological evidence of benign disease abutting chondrosarcoma

${ }^{\mathrm{b}}$ Tissue at first diagnosis not available for review

${ }^{c}$ Loss of two copies of $C D K N 2 A$ in the presence of one or two centromeric signals rearrangement involving either one or both genes. The genes involved in the rearrangements by anatomical site are shown in Fig. 2.

Synovial chondrosarcoma was diagnosed in seven patients from whose samples were available for analysis representing 7\% (7/97) of the combined benign and malignant cohort. Four of the seven cases gave informative
FISH results: three of these showed an FNI and/or ACVRA2 gene rearrangement (Table 2).

In three of the seven cases (cases 1,2, and 7) synovial chondrosarcoma presented de novo, without a history of conventional synovial chondromatosis. The other (cases 3-6) had a history of benign synovial chondromatosis prior to the diagnosis of secondary chondrosarcoma (Fig. 3; Table 2). 
Fig. 3 Photomicrographs of four cases of synovial

chondrosarcomas: a, b (Case 2) Interface between the well differentiated and high-grade cellular components; b nuclear polymorphism and an open chromatin appearance in the high-grade component. FISH reveals homozygous deletion of CDKN2A. c, d (Case 3)

(c) Multilobular appearance characteristic of synovial chondromatosis, but with (d) large atypical cells exhibiting well-defined borders and an open chromatin pattern. e, $\mathbf{f}$ (Case 5). There is significant high-grade atypia and loss of lobularity. g, h (Case 6) Tumor lobularity is retained but there is high-grade cytological atypia. FISH for CDKN2A was not available for cases 3, 5, and 6
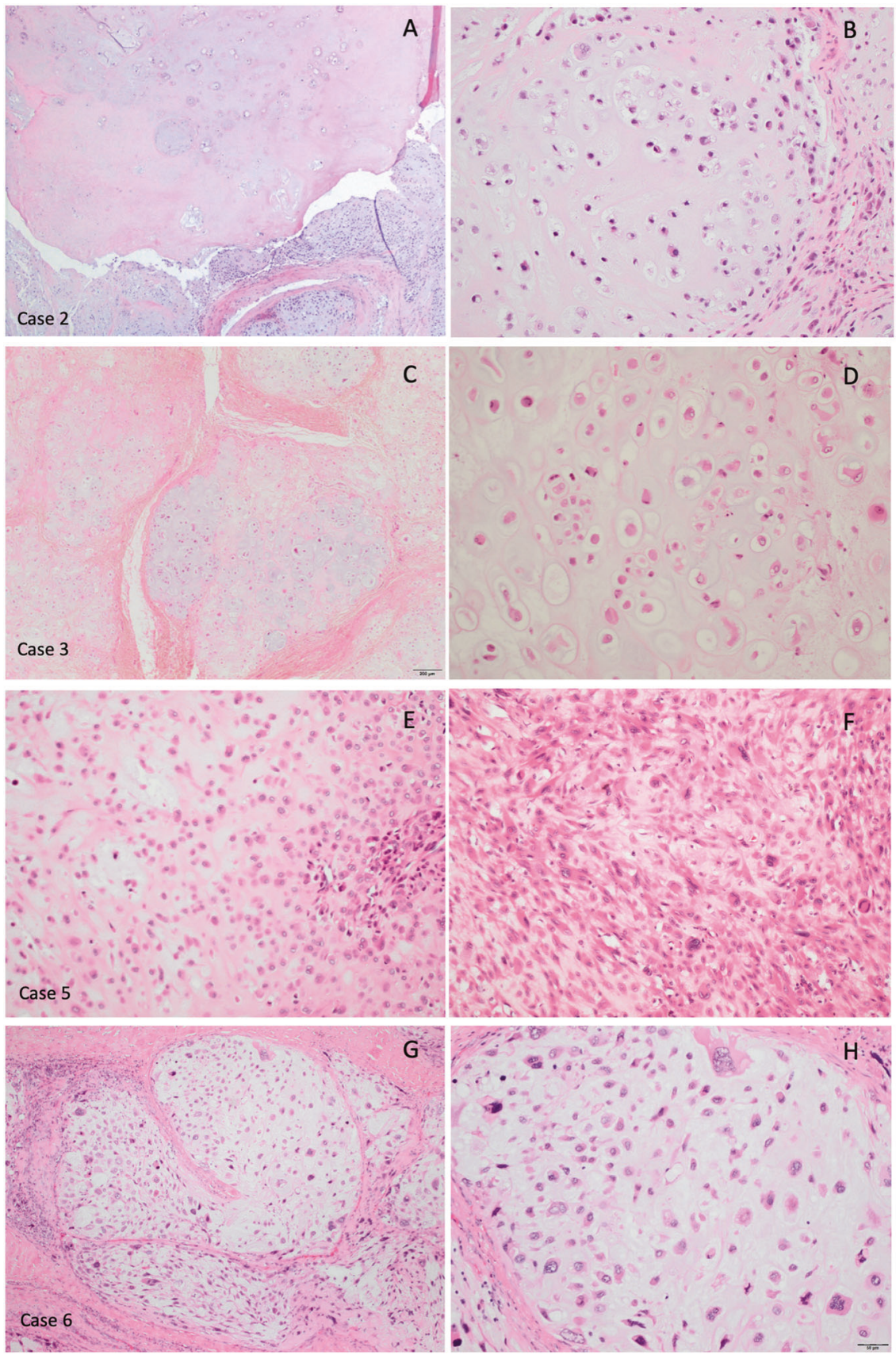

\section{CDKN2A copy number in synovial chondromatosis and secondary chondrosarcoma}

As $C D K N 2 A$ copy number alterations are commonly seen in high-grade conventional chondrosarcoma [17, 19], we investigated $C D K N 2 A$ copy number by FISH (Table 2). Four of the seven cases were informative: two showed homozygous deletion, one copy number gain, and one (case 3) showed a disomic pattern in the synovial chondromatosis component, but the area showing features of chondrosarcoma was not available for analysis (Table 2). Eighteen cases of synovial chondromatoses revealed disomy for $C N K N 2 A$.
Soft tissue chondroma samples were obtained from 19 patients $(10 \mathrm{~F}: 9 \mathrm{M})$ ranging from 15 to 83 years of age at presentation (mean 56.5 years). Nine tumors were sited in the foot, nine in the hand/wrist, and one in the forearm. Tumor size was available from 14 patients and ranged from 4 to $85 \mathrm{~mm}$ (mean $30 \mathrm{~mm}$ ). Clinical follow-up was available for eight patients: one tumor was not resected and the other seven have not recurred at the time of analysis (follow-up 0-19 months: mean 6 months).

Histologically, 12/19 soft tissue chondromas showed grungy matrix calcification, five of which exhibited chondroblastoma-like features [11] (Fig. 4). Two other 
Fig. 4 Soft tissue chondroma of the left third toe. a Dorsoplantar radiograph shows a calcified mass eroding the lateral aspect of the proximal phalanx of the third toe. Sagittal STIR (top) and T1 MR images show a lobular mass containing low signal foci consistent with punctuate calcification, eroding the adjacent bone. Axial STIR MR image (right) showing the hyperintense chondral tumor at the plantar aspect of the third toe (tumor indicated by arrows in all images). b Photomicrograph of a multinodular tumor with grungy-type and c pericellular calcification (chondroblastomalike). d FN1 gene rearrangement detected by FISH (break-apart signals). FGFR2 gene rearrangement detected by FISH (break-apart signals)

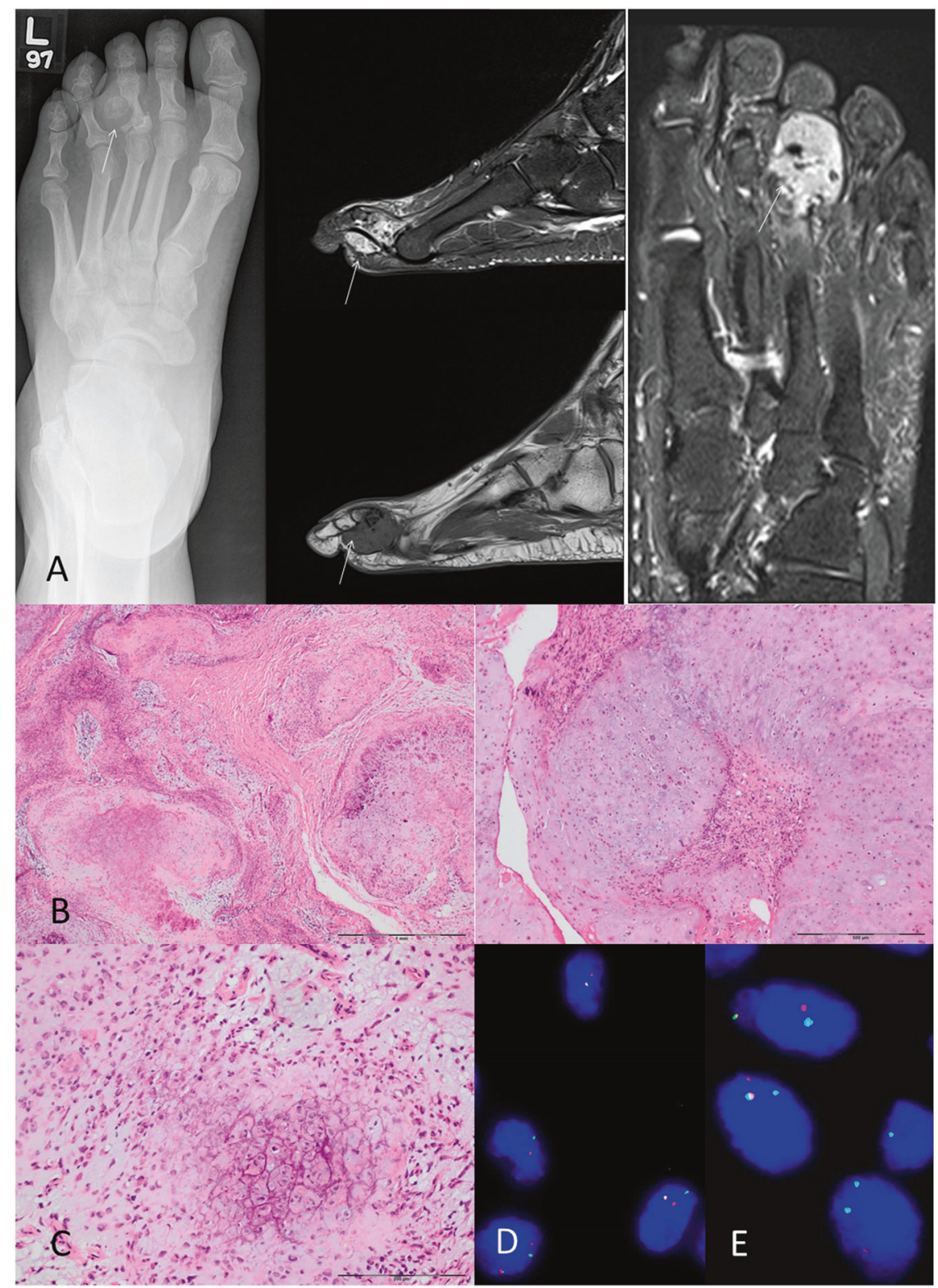

cases showed hyaline matrix without evidence of calcification, and the remaining five cases exhibited a predominantly myxoid cartilaginous matrix. Two of the cases with grungy calcification were associated with calcium pyrophosphate deposition disease.

Informative FISH results for $F N 1$ and $A C V R 2 A$ were obtained in $18 / 19$ cases. Nine $(50 \%)$ of the cases exhibited a FN1 gene rearrangement: all nine belonged to the group with grungy matrix calcification, three of which were classified as the chondroblastoma-like variant (Fig. 4). None of the cases with a predominantly myxoid or hyaline cartilaginous matrix without calcification showed a $F N 1$ gene rearrangement. ACVR $2 A$ rearrangements were not detected in any of the informative cases. Seven of the nine cases in which an FN1 gene rearrangement was detected were sited in the foot, the other two occurred in the hand.

In view of five cases showing features of chondroblastoma-like soft tissue chondroma, we analyzed these samples for H3.3 K36M expression, an alteration seen in $98 \%$ of conventional chondroblastomas [8]. None exhibited expression, thereby essentially excluding the possibility of $H 3 F 3 A / B \mathrm{~K} 36 \mathrm{M}$ mutation.

\section{RNA sequencing of synovial chondromatosis/ chondrosarcoma}

As $A C V R 2 A$ and/or $F N 1$ rearrangements were detected in $33 / 58(57 \%)$ cases of synovial chondromatosis and in $9 / 18$ $(50 \%)$ soft tissue chondromas we next sought other 

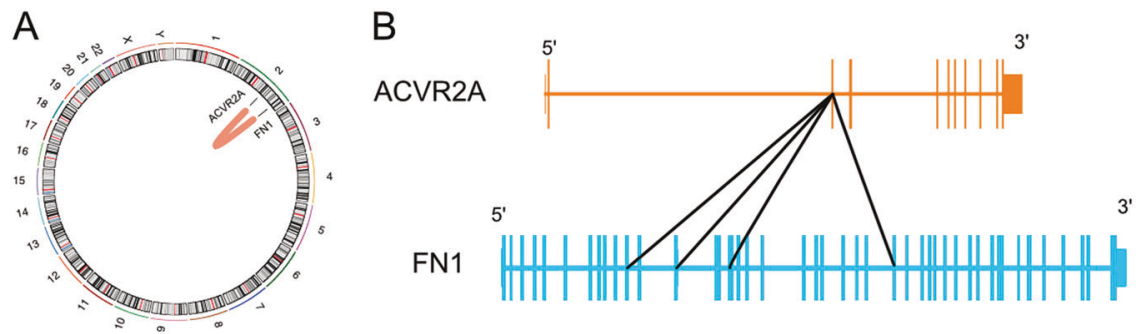

C

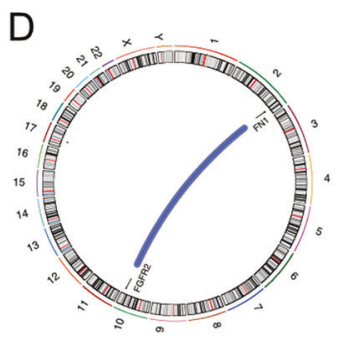

E

FGFR
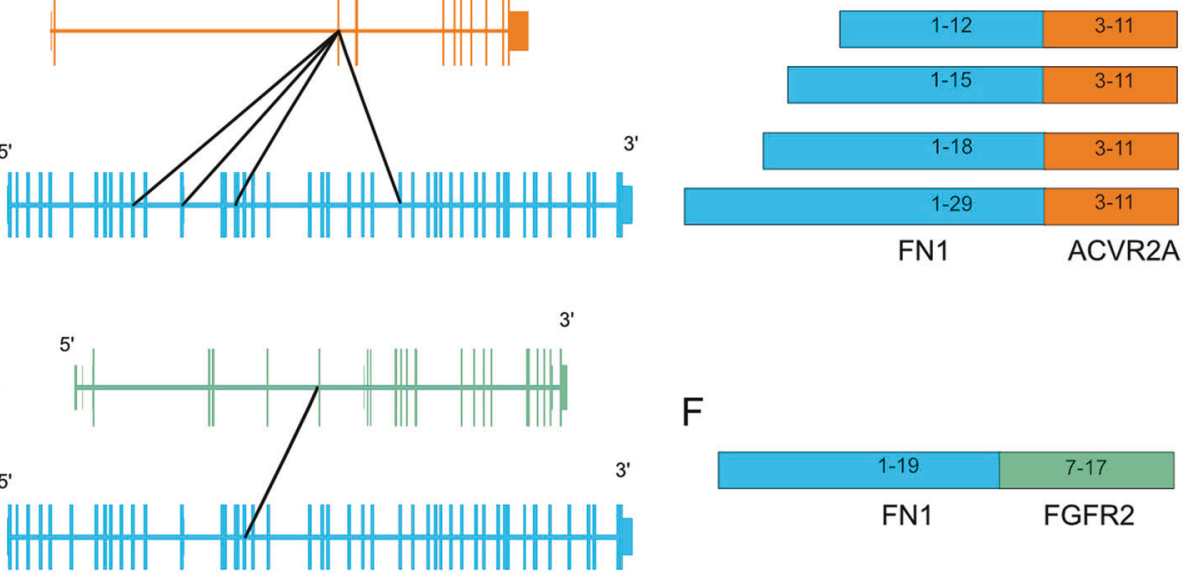

$\mathrm{F}$

FN1

Fig. 5 Fusion genes identified in synovial chondromatosis and soft tissue chondroma. a FN1-AVCR2A fusion shown in genomic context. b Exon map and c resulting exon composition of FN1-ACVR2A

fusions identified. d FN1 fusion with chromosome 10 genes $F G F R 2$, $C C D C 7$, and CRTAC1 in genomic context. e Exon map and $\mathbf{f}$ resulting exon composition of $F N 1-F G F R 2$ fusion

rearrangements by RNA sequencing. An $F N 1$ to $A C V R 2 A$ fusion, likely as the result of a $70 \mathrm{MB}$ inversion of chromosome 2, was detected in all three cases of synovial chondromatosis, and the single case of secondary chondrosarcoma studied (the whole exome of which has been reported previously) [20] (Fig. 5). As FN1 and AVCR2A are located on opposite genomic strands, the inversion resulted in a fusion in which both genes are in the same sense as each other and in every tumor in which the fusion was present, the predicted protein product was also in-frame. The location of the breakpoint within FNl was different in each tumor studied whereas the breakpoint in ACVR2A always occurred between the second and third exon. The chromosomal inversion therefore results in the entire protein-coding length of $A C V R 2 A$ being appended to a truncated N-terminus of FN1. In all four cases, a transcript representing the reciprocal arrangement with $A C V R 2 A$ at the $5^{\prime}$ end and $F N 1$ at the $3^{\prime}$ end was also formed, and the resulting fusion protein was predicted to be in-frame (Table S3).

Previous studies of synovial chondromatosis have revealed a number of karyotypic aberrations including abnormalities of chromosome 6 and a $(3 ; 14)$ translocation [3]. No direct evidence of these abnormalities was detected by RNA sequencing, however in the absence of a fusion gene being generated, RNA sequencing is not the modality of choice for investigating genomic structural variations.

\section{A novel recurrent FN1-FGFR2 fusion was identified in cases of soft tissue chondroma}

RNA sequencing of a soft tissue chondroma revealed a $(2 ; 10)$ translocation in which the $5^{\prime}$ end of $F N 1$ was fused to

FGFR2 (Fig. 5). Five different $F N 1-F G F R 2$ transcripts were detected in which only the $F N 1$ segment was constant whereas there were different $F G F R 2$ components. These different transcripts likely originated from a single genomic event and were resulted in alternative splicing of the premRNA. Only one of the observed FN1-FGFR2 transcripts formed an in-frame fusion that could potentially result in a functional chimeric protein. Additional fusions involving FNI and genes on chromosome 10 were also seen, although they were detected at much lower expression levels compared to $F N 1-F G F R 2$. A summary of the genetic changes observed in soft tissue chondroma is presented in supplementary Table 4.

\section{Extended study of FGFR2 rearrangements}

We confirmed the FGFR2 rearrangement detected on RNA sequencing using break-apart FISH probes. We next screened by FISH all cases of synovial chondromatosis, soft tissue chondroma, and synovial chondrosarcoma, in which break-apart signals were not detected for either ACVR $2 A$ and/or FN1: cases where only one of these genes was detected by FISH were also analyzed for the FGFR2 rearrangement. Of the 15 soft tissue chondroma cases tested, nine had an FNI rearrangement, and six were wild type for FN1 and ACVR2A. Remaining tissue for analysis was not available from three of the initial cohort tested (leaving 18 informative cases). Six of the nine cases with an FN1 gene rearrangement revealed additional $F G F R 2$ rearrangements, indirectly confirming an FN1-FGFR2 fusion (Table S4).

$F G F R 2$ gene rearrangements were not found in any case of synovial chondromatosis and synovial chondrosarcoma (Suppl Table S2). 


\section{Extended study of FGFR1 rearrangements}

Finally, driven by our impression that soft tissue chondroma with grungy calcification shared histological similarities with phosphaturic mesenchymal tumors, known to harbor FN1-FGFR1, and FN1-FGF1 fusion genes [9], we speculated that both genes represented candidate fusion genes partners in soft tissue chondromas. We screened the cases of soft tissue chondromas where tissue was available material $(n=15)$ for $F G F 1$ and $F G F R 1$ using break-apart FISH probes: in three cases an FGFRI gene rearrangement was detected. An FNI gene rearrangement was also identified in these three cases by FISH thereby indirectly confirming an FN1-FGFRI fusion in these cases. An FGF1 gene rearrangement was not observed in soft tissue chondromas. (Table S4). None of the cases of synovial chondromatosis $(n=18)$ and synovial chondrosarcoma $(n=1)$ where material was available exhibited either an $F G F 1$ or FGFRl gene rearrangement.

\section{FGF23 mRNA levels in soft tissue chondromas}

In view of an FN1-FGFRl fusion gene being characteristic of phosphaturic mesenchymal tumor and typically expressing high levels of $F G F 23$ we investigated this using RNA in situ hybridization (RNAscope) and qPCR in eight soft tissue chondromas cases showing an FNI break-apart signal of our series. None of the cases studied revealed significant levels of FGF23 by either test (Supplementary Fig. 1).

\section{Discussion}

The current study demonstrates that at least $57 \%$ of synovial chondromatosis cases show a rearrangement involving $F N 1$ and/or $A C V R 2 A$ as detected by FISH. The true prevalence of cases harboring these alterations is likely to be higher on the basis that only one of three synovial chondroma/chondromatosis cases that revealed an FN1-ACVR2A fusion gene by RNA sequencing demonstrated break-apart signals involving both genes. This discrepancy could be explained by the proximity of both genes on chromosome 2 and the limitation of designing appropriate FISH probes to detect such a paracentric inversion event. The one case, which was not informative for $F N 1$ on FISH highlights the finding that possibly around $30 \%$ of cases are noninformative by FISH, demonstrating the need to improve tissue processing of calcified samples.

We have also confirmed the previously reported occurrence of an FN1 and $A C V R 2 A$ gene rearrangement in the malignant variant of synovial chondromatosis [2]. As $7 \%$ of our cohort was represented by synovial chondrosarcoma our data support previous reports that $\sim 10 \%$ of synovial chondromatosis undergo malignant transformation [21, 22]. The presence of an FN1-ACVR2 fusion did not distinguish between benign and malignant disease, but we showed that copy number alteration of $C D K N 2 A$ appears to be a valuable predictor of malignancy, similar to conventional cartilaginous tumors $[17,20]$ where copy number alterations of $C D K N 2 A$ appear exclusively in chondrosarcomas.

We report for the first time that soft tissue chondromas harbor recurrent $F N 1-F G F R 1$ and $F N 1-F G F R 2$ gene fusions demonstrating conclusively that they represent neoplasms. The mutual exclusivity in detection of $F G F R I$ and $F G F R 2$ fusion genes in soft tissue chondromas and $A C V R 2 A$ fusions in synovial chondromatosis is supportive of them being distinct pathological entities [13, 14]. The ability to distinguish reliably between these two entities is clinically relevant since soft tissue chondromas have a very low risk of local recurrence, and malignant transformation has not yet been described. As a consequence, they can be managed more conservatively than synovial chondromatosis.

Remarkably we found no difference in the histological appearance between synovial chondromatosis with and without $F N 1-A C V R 2 A$ rearrangements. In contrast, all the soft tissue chondromas harboring $F N 1$ alterations exhibited 'grungy calcification', a finding that in some cases is reminiscent of chondroblastomas, and led to the description of the chondroblastoma-like variant [11]. Phosphaturic mesenchymal tumors typically also show this particular type of mineralization and are characterized by FNIFGFR1 fusions in $42 \%$ of cases [9], and it was this which prompted us to test our set of soft tissue chondromas for FGFR1 alterations. However, in contrast to phosphaturic mesenchymal tumors in which FGF23 expression is detected in $\sim 95 \%$ of cases [23], we did not detect high levels of FGF23 mRNA expression in the soft tissue chondromas. Further study is needed to understand the distinct biological consequences of FN1-FGFRl fusions in these tumor types and to explain the expression of FGF23 in PMT but not soft tissue chondroma. The presence of the same translocation in two tumors of mesenchymal origin is suggestive that there exists some commonality in the pathogenesis of both entities. Explanations for the difference between the two tumor types could reside in different cells of origin in which the translocation occurred or in the epigenetic composition of the cells. Irrespective of the mechanism, we consider that it would be prudent to measure the serum phosphate levels in patients whose tumors harbor an FN1 fusion to ensure that any metabolic disturbances can be identified and addressed. Soft tissue chondromas without FNl rearrangements exhibited a distinctive myxochondroid morphology and might represent a different entity. Indeed, it may be that these cases represent a result of a degenerative/reparative process. 
In the single case of soft tissue chondroma that was subjected to RNA sequencing, there were several other gene fusions involving chromosome 10 resulting in predicted inframe fusions with both FGFR2 and CRTAC1, in addition to the $F N 1-F G F R 2$ fusion. Fusions being formed with two genes in close proximity to each other on chromosome 10 cannot be explained by a simple single translocation and imply that further genomic rearrangements of the region have also occurred.

Of the four synovial chondromatosis/synovial chondrosarcoma cases subjected to RNA sequencing in our study, all revealed the presence of fusions involving $F N 1$ and $A C V R 2 A$ as previously demonstrated, thereby supporting the findings in the larger number of our cases probed by FISH. However, it is not possible to state which of these structurally altered genes drives tumor progression as both $F N 1-A C V R 2 A$ and $A C V R 2 A-F N 1$ are predicted to be in-frame. Both $F N 1$ and $A C V R 2 A$ have been implicated in the development of neoplasia. ACVR2A encodes a receptor to Activin A, a protein that is part of the large family of transforming growth factor $\mathrm{B}$ genes and is involved in regulating multiple cellular processes. ACVR2A is commonly mutated in colorectal carcinoma and as well as hepatocellular and cholangiocarcinoma [24, 25]. FN1 is involved in a number of cell processes including adhesion and migration, the aberrant regulation of which could contribute to a neoplastic process. In addition to phosphaturic mesenchymal tumors discussed above, FN1 fusions with $A L K$ have been described in gastrointestinal leiomyomas [26], and $E G F$ fusions in calcifying aponeurotic fibromas [27].

In terms of explaining the mechanism of action of the fusion genes in synovial chondromatosis and soft tissue chondromas, it is noteworthy that $F N 1$ is found as the $5^{\prime}$ fusion partner of both $A C V R 2 A$ and FGFR2, and that the $3^{\prime}$ fusion partners have not been found with other $5^{\prime}$ partners. This implies that the FNI component of the fusion plays an important biological role in the pathogenesis of these tumors and is not acting simply through driving the expression of the $3^{\prime}$ partner through an active promoter. The FN1-FGFR2 fusion identified by RNA sequencing in the soft tissue chondroma in our study reveals a similar domain structure of the resultant proteins to that found previously involving the $F N 1-F G F R 1$ fusion in phosphaturic mesenchymal tumor [9]. In both cases, the N-terminal region of FN1 is attached to a truncated extracellular region of an FGF receptor leaving the transmembrane and intracellular domains intact. As previously hypothesized by Lee et al., the presence of the N-terminal region of FN1 may aid the dimerization of the fused FGF receptor through the fibronectin domain leading to signal activation [9]. This same mechanism may explain the action of the FNI$A C V R 2 A$ fusion, namely that $F N 1$ may facilitate dimerization of $A C V R 2 A$ inducing activin signaling. In those cases in which FISH did not identify a fusion, it is possible that activation of the FGF pathway has been achieved through other mechanisms or that the disease is being driven by a mechanism that have yet to be discovered.

Acknowledgements This research was funded by a grant to LPC by The Pathological Society of Great Britain and Ireland, the RNOH NHS Trust R\&D Office, and Skeletal Action Trust. The Bone Cancer Research Trust provided funding for biobanking to AMF and PC. AMF is a NIHR senior investigator and is supported by the National Institute for Health Research, UCLH Biomedical Research Centre, and the UCL Experimental Cancer Centre. MR is funded by the Tom Prince Cancer Trust. NP is a Cancer Research UK-funded clinician scientist. We are grateful to the RNOH Musculoskeletal Pathology Biobank team for consenting patients and accessing samples, to the UCL Cancer Research UK Cancer Centre core Sequencing and Pathology facilities. We thank patients for participating in our research and the clinical teams involved in their care.

\section{Compliance with ethical standards}

Conflict of interest The authors declare that they have no conflict of interest.

Publisher's note: Springer Nature remains neutral with regard to jurisdictional claims in published maps and institutional affiliations.

\section{References}

1. Walker EA, Murphey MD, Fetsch JF. Imaging characteristics of tenosynovial and bursal chondromatosis. Skelet Radiol. 2011;40:317-25.

2. Totoki Y, Yoshida A, Hosoda F, Nakamura H, Hama N, Ogura K, et al. Unique mutation portraits and frequent COL2A1 gene alteration in chondrosarcoma. Genome Res. 2014;24:1411-20.

3. Buddingh EP, Krallman P, Neff JR, Nelson M, Liu J, Bridge JA. Chromosome 6 abnormalities are recurrent in synovial chondromatosis. Cancer Genet Cytogenet. 2003;140:18-22.

4. Mertens F, Jonsson K, Willen H, Rydholm A, Kreicbergs A, Eriksson L, et al. Chromosome rearrangements in synovial chondromatous lesions. Br J Cancer. 1996;74:251-4.

5. Sciot R, Dal Cin P, Bellemans J, Samson I, Van den Berghe H, Van Damme B. Synovial chondromatosis: clonal chromosome changes provide further evidence for a neoplastic disorder. Virchows Arch. 1998;433:189-91.

6. Tallini G, Dorfman H, Brys P, Dal Cin P, De Wever I, Fletcher $\mathrm{CD}$, et al. Correlation between clinicopathological features and karyotype in 100 cartilaginous and chordoid tumours. A report from the chromosomes and morphology (CHAMP) collaborative study group. J Pathol. 2002;196:194-203.

7. Amary MF, Bacsi K, Maggiani F, Damato S, Halai D, Berisha F, et al. IDH1 and IDH2 mutations are frequent events in central chondrosarcoma and central and periosteal chondromas but not in other mesenchymal tumours. J Pathol. 2011;224:334-43.

8. Amary MF, Berisha F, Mozela R, Gibbons R, Guttridge A, O'Donnell P, et al. The H3F3 K36M mutant antibody is a sensitive and specific marker for the diagnosis of chondroblastoma. Histopathology. 2016;69:121-7.

9. Lee JC, Su SY, Changou CA, Yang RS, Tsai KS, Collins MT, et al. Characterization of FN1-FGFR1 and novel FN1-FGF1 fusion genes in a large series of phosphaturic mesenchymal tumors. Mod Pathol. 2016;29:1335-46. 
10. Wang L, Motoi T, Khanin R, Olshen A, Mertens F, Bridge J, et al. Identification of a novel, recurrent HEY1-NCOA2 fusion in mesenchymal chondrosarcoma based on a genome-wide screen of exon-level expression data. Genes Chromosomes Cancer. 2012; 51:127-39.

11. Cates JM, Rosenberg AE, O'Connell JX, Nielsen GP. Chondroblastoma-like chondroma of soft tissue: an underrecognized variant and its differential diagnosis. Am J Surg Pathol. 2001;25:661-6.

12. Lichtenstein L, Goldman RL. Cartilage tumors in soft tissues, particularly in the hand and foot. Cancer. 1964;17:1203-8.

13. Sciot R, Bridge JA. Synovial chondromatosis. In: Fletcher CDM, Bridge JA, Hogendoorn PC, editors. WHO classification of tumours of soft tissue and bone. Lyon, France: IARC Press; 2013. p. 261.

14. Rosenberg AE, Mandahl N. Soft-tissue chondroma. In: Fletcher CDM, Bridge JA, Hogendoorn PC, editors. WHO classification of tumours of soft tissue and bone. Lyon, France: IARC Press; 2013. p. $160-1$.

15. Damato S, Alorjani M, Bonar F, McCarthy SW, Cannon SR, O'Donnell P, et al. IDH1 mutations are not found in cartilaginous tumours other than central and periosteal chondrosarcomas and enchondromas. Histopathology. 2012;60:363-5.

16. Amary MF, Berisha F, Bernardi Fdel C, Herbert A, James M, Reis-Filho JS, et al. Detection of SS18-SSX fusion transcripts in formalin-fixed paraffin-embedded neoplasms: analysis of conventional RT-PCR, qRT-PCR and dual color FISH as diagnostic tools for synovial sarcoma. Mod Pathol. 2007;20:482-96.

17. Amary MF, Ye H, Forbes G, Damato S, Maggiani F, Pollock R, et al. Isocitrate dehydrogenase 1 mutations (IDH1) and p16/ CDKN2A copy number change in conventional chondrosarcomas. Virchows Arch. 2015;466:217-22.
18. Nicorici D, Satalan M, Edgren H, Kangaspeska S, Murumagi A, Kallioniemi O, et al. FusionCatcher-a tool for finding somatic fusion genes in paired-end RNA-sequencing data. bioRxiv 2014:011650.

19. Schrage YM, Lam S, Jochemsen AG, Cleton-Jansen AM, Taminiau AH, Hogendoorn PC, et al. Central chondrosarcoma progression is associated with $\mathrm{pRb}$ pathway alterations: CDK4 downregulation and p16 overexpression inhibit cell growth in vitro. J Cell Mol Med. 2009;13:2843-52.

20. Tarpey PS, Behjati S, Cooke SL, Van Loo P, Wedge DC, Pillay $\mathrm{N}$, et al. Frequent mutation of the major cartilage collagen gene COL2A1 in chondrosarcoma. Nat Genet. 2013;45:923-6.

21. Bertoni F, Unni KK, Beabout JW, Sim FH. Chondrosarcomas of the synovium. Cancer. 1991;67:155-62.

22. Biazzo A, Confalonieri N. Synovial chondrosarcoma. Ann Transl Med. 2016;4:280.

23. Carter JM, Caron BL, Dogan A, Folpe AL. A novel chromogenic in situ hybridization assay for FGF23 mRNA in phosphaturic mesenchymal tumors. Am J Surg Pathol. 2015;39:75-83.

24. Yuza K, Nagahashi M, Watanabe S, Takabe K, Wakai T. Hypermutation and microsatellite instability in gastrointestinal cancers. Oncotarget. 2017;8:112103-15.

25. Wang A, Wu L, Lin J, Han L, Bian J, Wu Y, et al. Whole-exome sequencing reveals the origin and evolution of hepatocholangiocarcinoma. Nat Commun. 2018;9:894.

26. Panagopoulos I, Gorunova L, Lund-Iversen M, Lobmaier I, Bjerkehagen B, Heim S. Recurrent fusion of the genes FN1 and ALK in gastrointestinal leiomyomas. Mod Pathol. 2016;29: 1415-23.

27. Puls F, Hofvander J, Magnusson L, Nilsson J, Haywood E, Sumathi VP, et al. FN1-EGF gene fusions are recurrent in calcifying aponeurotic fibroma. J Pathol. 2016;238:502-7. 